\title{
Semantic Asymmetries must Originate in a Visual Asymmetry
}

\author{
Carol Whitney \\ Department of Linguistics \\ University of Maryland, College Park \\ cwhitney@cs.umd.edu
}

\begin{abstract}
Many asymmetries have been observed in half-field studies of visual word recognition. I argue that any such asymmetries must originate at a pre-lexical level. The SERIOL model of orthographic encoding explains how and why an asymmetry arises in visual processing of letter strings. This theory has allowed the reversal of asymmetries related to string length and to orthographic-neighborhood size. In this chapter, I examine the issue of how such visual processing could explain asymmetries associated with semantic priming and N400 amplitude.
\end{abstract}




\section{Introduction}

Visual half-field studies, where stimuli are presented to the right or left parafovea, are widely used in the investigation of visual word recognition and semantic processing. Due to the architecture of the visual system, information from the left visual field (LVF) initially projects to the right hemisphere $(\mathrm{RH})$, while the right visual field (RVF) projects to the left hemisphere (LH). Therefore, observed asymmetries in half-field studies have been used to draw conclusions about specializations of the cerebral hemispheres. For example, based on lateralized studies of semantic priming, Burgess and Simpson (1988) said: "Results suggest that, while automatic processing occurs in both hemispheres, only the left hemisphere engages in controlled processing of ambiguous word meanings. In addition, the present results support the idea that the right hemisphere has a special role in ambiguity resolution and that the right hemisphere lexicon possesses a richer endowment than earlier thought." Also based on semantic priming, Koivisto and Laine (2000) said: "It is suggested that the left hemisphere automatically activates categorically related word meanings in both hemispheres. The right hemisphere contributes by maintaining the meanings active and by retrospectively integrating them to the context." Based on asymmetric N400 patterns in a lateralized ERP study, Kutas and Federmeier (1999) said: "We propose that right hemisphere processing is best characterized as 'integrative'; new information is compared directly with context information. In contrast, left hemisphere processing is better characterized as 'predictive'; the processing of context leads to an expectation about the semantic features of upcoming items and new information is compared with that expectation rather than directly with the context."

Such interpretations of hemispheric specialization are based on the underlying assumption that processing remains dominated by the hemisphere contralateral to the stimulus. That is, LVF presentation invokes $\mathrm{RH}$-specific linguistic mechanisms, while RVF presentation invokes LH-specific linguistic mechanisms. However, this widespread assumption is inconsistent with the results of brain-imaging experiments. MEG and fMRI studies have shown that for central presentation, processing becomes lateralized to the left fusiform gyrus, where a retinotopic representation of the string is converted into a abstract encoding of letter order in the Visual Word Form Area (VWFA) (Dehaene, Jobert, Naccache, Ciuciu, Poline, Le Bihan, and Cohen, 2004; McCandliss, Cohen \& 
Deheane, 2003; Tarkiainen, Helenius, Hansen, Cornelissen \& Salmelin, 1999). This left lateralization is present even for half-field presentation. That is, brain activation patterns are identical for LVF and RVF presentation at the level of the VWFA and beyond (Cohen, Dehaene, Naccache, Lehericy, Dehaene-Lambertz, Henaff, \& Michel, 2000). Furthermore, patients suffering from isolated lesions to the splenium of the corpus callosum show pure alexia for words presented to the $\mathrm{LVF} / \mathrm{RH}$, but unimpaired reading for words presented to the RVF/LH (Cohen, Martinaud, Lemer, Lehericy, Samson, Obadia, Slachevsky, \& Dehaene, 2003). This pattern of deficit shows that orthographic information going to the LVF/RH is normally transferred to the $\mathrm{LH}$ prior to lexical access. In sum, these studies indicate that an abstract encoding of letter order is created in the $\mathrm{LH}$, and lexical access occurs in the $\mathrm{LH}$, independently of presentation location. Therefore, processing at the lexical levels and above must be qualitatively the same for RVF or LVF presentation. See Figure 1.

\section{Figure 1 about here.}

If so, $\mathrm{VF}$ asymmetries must actually originate a level of processing prior to this $\mathrm{LH}$ convergence. However, these are visual areas. How could visual processing create asymmetries in performance of lexical and semantic tasks? Based on the SERIOL model of the lexical route of visual word recognition, I have proposed that hemisphere-specific visual processing is required to form the abstract encoding of letter order, and that such processing leads to VF-specific activation patterns at the pre-lexical level (Whitney, 2001). This difference in activation patterns affects lexical acccess, giving the appearance of qualitatively different performance patterns at the lexical level (Whitney, 2004). We (Whitney \& Lavidor, 2004; 2005) have verified this account for asymmetries related to length and orthographic-neighborhood size $(\mathrm{N})$, by using contrast adjustments to manipulate activation patterns, resulting in the reversal of the normal asymmetries.

Our experimental work thus far has focused on lexical properties (length and $\mathrm{N}$ ). Semantic asymmetries would also have to originate at the visual level, but it less obvious how activation patterns could affect this higher level of processing. This issue is the main focus of the present chapter. The organization of this chapter is as follows. First I review the SERIOL model and the results of the length and $\mathrm{N}$ experiments. Then I address VF effects in semantic priming, and on N400 amplitude in the above ERP study (Kutas \& 
Federmeier, 1999). The chapter concludes with a discussion of the implications of this work, and brief comments on directions for future research.

\section{SERIOL Model}

It is well known that neural representations increase in abstractness as distance from the periphery increases. The SERIOL model offers a comprehensive theory of the representational transformations carried out by a skilled reader in the processing stream extending from primary visual cortex to lexical access (along the orthographic, lexical route). I first give an overview of the theory, and then present the levels relevant VF asymmetries in more detail. For brevity, I do not include supporting experimental evidence for the model; such arguments can be found elsewhere (Whitney, 2001a; 2001b; 2002; Whitney \& Berndt, 1999).

\section{Overview}

The SERIOL framework is comprised of five layers: edge, feature, letter, bigram, and word. Each layer is comprised of nodes, corresponding to neural assemblies. For the edge, feature, and letter layers, a letter's activation is taken to be the total amount of neural activity devoted to representing that letter. Thus a letter's activation increases with number the nodes activated by the letter, and their firing rate and firing duration.

The edge layer corresponds to the earliest levels of visual processing $(\mathrm{V} 1, \mathrm{~V} 2)$, where the representation of the string is split across the hemispheres, and receptive fields are small. Here there is an activation pattern resulting from the acuity gradient. This gradient originates in the density of cones in the retina and is magnified into the cortex such that the amount of cortical area representing a fixed amount of visual space is highest at fixation and falls off as eccentricity increases. Thus, in the model, letter activations are highest near fixation, and fall off as distance from fixation increases.

At the feature layer (V4, posterior fusiform), units are more broadly tuned to retinal location, while the representation of the string is still split across the hemispheres. As discussed in more detail below, I proposed that the acuity gradient is converted into an activation pattern, dubbed the locational gradient, in which activation level decreases across the string from left to right. For example, for the stimulus BIRD, B's features 
become the most highly activated, I's the next most activated, R's the next, and D's the least.

The letter layer (LH fusiform posterior to the VWFA) is comprised of nodes that synchronously oscillate in excitability (Hopfield, 1995; Lisman \& Idiart, 1995). The interaction of the locational gradient with the variations in excitability induces sequential firing. That is, timing of firing depends on the amount of input to a letter node; a letter node receiving more input fires before one receiving less input. In our example, B fires, then I, then R, and then D. This conversion also results in varying letter activation levels. In general, letter activations are similar to their feature activations, except that activation increases at the final letter because its firing is not inhibited by a subsequent letter (Whitney \& Berndt, 1999; Whitney, 2001a), as is consistent with the well-known finalletter advantage.

It is assumed that this serial representation of letter order serves separate lexical and phonological routes to the lexicon. The model focuses on the lexical route. The bigram layer (VWFA) is comprised of nodes that represent ordered letter pairs. That is, a bigram node is activated when its constituent letters fire in a certain order. Thus, bigram nodes $\mathrm{BI}$, IR, and RD, as well as those corresponding to non-contiguous letter pairs (BR, BD, and ID), become activated in our example. A bigram node's activation level depends on the time lag between the firing of its constituent letters; bigram nodes activated by contiguous letters become more highly activated than those activated by non-contiguous letters.

Bigram nodes then activate the word layer (LH temporal areas) via weighted connections that record the bigram activation pattern resulting from each word. The input to a word unit is calculated in the usual way, as the dot-product of the input and weight vectors. Lateral inhibition within the word layer allows the most highly activated word node to become the winner.

In summary, the acuity gradient at the edge layer is transformed into the monotonically decreasing locational gradient at the feature layer. The locational gradient is converted into a serial encoding at the letter layer, which is then decoded into a set of ordered letter pairs at the bigram layer. Bigram nodes contact the word layer via weighted connections. Thus the model specifies how the initial retinotopic representation is 
transformed into a location-invariant (serial) representation of letter order, and how this representation activates the word layer via a pre-lexical (bigram) encoding that is based on the relationship between letters, not on their absolute string position (Grainger \& Whitney, 2004).

As discussed above, VF asymmetries must arise prior to the lateralized LH representation, namely in the edge and feature layers of the model. Indeed, creation of the locational gradient requires hemisphere-specific processing, as discussed next.

\section{Locational gradient formation}

The acuity gradient is converted into the locational gradient as edge nodes activate feature nodes. Note that the proposed locational gradient monotonically decreases from the first letter to the last letter, whereas the acuity gradient increases from the first letter to the fixated letter (i.e., in the $\mathrm{LVF} / \mathrm{RH}$ ), and decreases from the fixated letter to the last letter (i.e., in the RVF/LH). See Figure 2. Therefore, the slope of the RH acuity gradient is in the opposite direction as required for the locational gradient, while the slope of the LH acuity gradient is in the same direction. Thus, in the RH, the acuity gradient's slope must be inverted as features are activated. In contrast, the acuity gradient's slope can be maintained as features are activated in the LH. As a result, processing at the feature layer differs across hemispheres, with the RH performing a more extensive transformation. This hemisphere-specific processing is learned during reading acquisition, perhaps in response to a top-down attentional gradient.

Figure 2 about here.

First we consider the $\mathrm{RH}$ processing. I propose that $\mathrm{RH}$ features become more highly activated by edge-level inputs than LH features, allowing the first letter's features to reach a high level of activation even if they are far from fixation. This could occur either via stronger learned connections from the edge to feature layers in the $\mathrm{RH}$, or via stronger self-excitation within the RH feature layer. Within the RH feature layer, strong directional lateral inhibitory connections are learned, such that nodes inhibit other nodes having receptive-field locations to its right. Thus, inhibitory input increases as letter

position increases, because more and more features send inhibition from the left. For example, consider the word BUSTLE as in Figure 2. B's features would input U's features, while B and U's features would inhibit S's features. This strong directional 
inhibition overrides the slope of the acuity gradient, inverting it. So the features comprising the first letter attain a high level of activation (due to strong excitation and lack of lateral inhibition), and activation decreases towards fixation (due to sharply increasing lateral inhibition from the letters to the left).

In the LH, excitatory and lateral inhibitory connections are weaker, because the acuity gradient's slope is already in the correct direction. Thus the acuity gradient is essentially maintained at the feature layer, although some directional inhibition may steepen its slope.

In addition to inhibition within hemispheres, there is also inhibition across hemispheres. The RH features inhibit the LH features, bringing the LH feature activations lower than those of the RH. Thus the two halves of the locational gradient are meshed to create a strictly decreasing activation gradient from the first to the last letter.

In summary, hemisphere-specific processing is required to form the locational gradient, due to differing acuity patterns across the visual fields. In the $\mathrm{RH}$, strong excitation and left-to-right lateral inhibition invert the acuity gradient to form the locational gradient. In the LH, the acuity gradient serves as the locational gradient.

\section{Parafoveal processing}

The hemisphere-specific transformations that are required to create the locational gradient for a fixated word have ramifications for parafoveally presented words. Next we focus on the resulting activation patterns.

Note that the proposed stronger bottom-up activation to the RH implies that an initial letter should be better perceived in the LVF than the RVF, even if it is at a larger eccentricity in the LVF. This is indeed the case (Bouma, 1973; Estes, Allmeyer \& Reder, 1976). In contrast, a second or third letter at a given eccentricity is less well perceived in the LVF than the RVF (Wolford \& Hollingsworth, 1974). This is explained by the strong LVF/RH left-to-right inhibition, indicating that this inhibition outweighs the effect of stronger bottom-up input. Moreover, mathematical modeling (Whitney, 2001) has shown that the theory of locational gradient formation explains detailed patterns of perceptibility in the interaction of eccentricity, visual field, and string position (Wolford \& Hollingsworth, 1974). 
These data and the modeling indicate the following activation feature-level patterns for half-field presentation. In the LVF/RH, strong inhibition from the first letter to the low-acuity second and third letters makes their activations quite low. However, as acuity increases for the final letters, lateral inhibition becomes insufficient, and their activations remain too high. Thus there is a sharp decrease in activation across the early string positions, and then a flattening across the final letters. See Figure 3. In contrast, the locational gradient remains smoothly decreasing for RVF presentation, because it is largely based on the acuity gradient.

Figure 3 about here.

I propose that such differing activation patterns are the source of VF asymmetries. Recall that bigram activation levels depend on the firing patterns of the constituent letters, which depend on the locational gradient. It is assumed that learned bigram-toword connection weights record the pattern of bigram activations for each word. Thus, the amount of bottom up input reaching a word node depends on how well the bigram activation vector "matches" the learned weight vector. (More precisely, it depends on the angle between the activation and weight vectors, as well as the magnitude of those vectors.) When the LVF locational gradient is not smoothly decreasing, it will create a bigram activation pattern that differs from the learned weight vector (which presumably is based on the activation pattern resulting from a smoothly decreasing gradient). Therefore, the bottom-up pattern does not match the learned pattern as well as for central or RVF presentation, reducing the amount of input to the corresponding word node.

\section{Length Experiment}

It is well known that there is a length effect in lexical decision for LVF, but not RVF, presentation (Ellis \& Young, 1988; Young \& Ellis, 1985). That is, reaction times (RTs) increase with string length for LVF presentation, but are independent of string length for RVF presentation (and central presentation, as well). As string length increases in the LVF, the locational gradient would become more and more non-smooth, increasing the mismatch with the learned weight vector, and creating a length effect. (See Whitney and Lavidor (2004) for a discussion of how a length effect could be absent for RVF and central presentation, despite serial processing at the letter level.) 
This analysis suggests that it should be possible to abolish the LVF length effect by "fixing" the locational gradient (i.e., making it smoother). Therefore, we (Whitney \& Lavidor, 2004) performed an experiment using position-specific contrast adjustments to manipulate bottom-up activation patterns. In the control condition, all letters were presented in light gray on a black background. In the adjust condition, the contrast of the second and third letters was increased by presenting those letters in white, and the contrast of the sixth letter (if present) was decreased by presenting it in medium gray, while letters at other positions remained light gray. Such an adjustment should smooth the gradient in the LVF as shown in Figure 4, thereby negating the length effect via facilitation for longer words (as compared to the control condition). Conversely, the same manipulation should degrade an otherwise smooth RVF gradient, creating a length effect. These predictions were confirmed, as shown in Figure 5. The LVF length effect was abolished, demonstrating that a length effect is not an inherent attribute of LVF/RH processing. Therefore, the asymmetry of the length effect does not arise from hemisphere-specific modes of lexical access (Ellis \& Young, 1988; Young \& Ellis; 1985), but rather originates in activation patterns at the visual level.

\section{Figures 4 and 5 about here.}

\section{$N$ experiment}

Another VF asymmetry is related to the $\mathrm{N}$ effect. $\mathrm{N}$ refers to the number of orthographic neighbors, words that can be formed by changing one letter of the target word (Coltheart, Davelaar, Jonasson, \& Besner, 1977). For example, BAND is a high-N word, with neighbors LAND, SAND, BEND, BIND, BARD, BALD, BANE, etc., while ONYX is a low-N word, with no neighbors. For CVF presentation of four-letter, lowfrequency words under lexical decision, high-N words are responded to more quickly than low-N words (Andrews, 1997). Interestingly, this $\mathrm{N}$ effect is present for LVF, but not RVF, presentation (Lavidor \& Ellis, 2002a,b). Note that this asymmetry goes in the opposite direction as the length effect; CVF presentation patterns with the LVF, not the RVF, indicating that the N-effect asymmetry cannot be attributed to less efficient LVF processing.

Although the SERIOL model focuses on bottom-up processing, this is not to imply an absence of top-down processing, To explain the $\mathrm{N}$ effect, I assume that there is 
feedback from the word layer to letter layer, and that lexical decision requires multiple oscillatory cycles (i.e., the sequence of letters fires multiple times). After the first oscillatory cycle, the activated word nodes then provide top-down input to the letter nodes. This feedback is also in the form a gradient, with the first letter receiving the most input, the second letter receiving the next most, etc. So during the second oscillatory cycle, letter nodes receive both bottom-up input (from the feature level) and top-down input (from the word level).

Input levels to letter nodes directly affect the temporal evolution of word node activations. Because letters are activated sequentially, bigrams are activated sequentially. Increased input to letter nodes would speed their firing, and the firing of their respective bigram nodes. This would speed the activation of the target word node, and decrease the amount of time that the target word node receives inhibition from competitor word nodes (Whitney, 2004), decreasing reaction times. Thus additional, top-down input from orthographic neighbors could speed letter activations and decrease reaction times.

In English, most orthographic neighbors match the target word's body. For many four-letter words, this would correspond to the last three letters. Therefore, the second, third and fourth letter nodes preferentially receive top-down input in the case of high-N. Because the top-down input is in the form of a gradient, the second and third letters receive more top-down input than the fourth letter, so the internal letters are the most strongly affected by high-N. For LVF presentation, this top-down activation gradient may also have the effect of smoothing the locational gradient across the last three letters, as the activation of the fourth letter's features may be relatively too high. This smoothing would also contribute to a facilitatory effect.

Why then is there no $\mathrm{N}$ effect for RVF presentation? Recall that the RVF locational gradient is based on the acuity gradient. It has been established that the acuity gradient becomes less steep as eccentricity increases (Westheimer, 1987). (While this seems contrary to the usual wisdom that "acuity falls off quickly outside the fovea", it is actually the case that acuity falls off most quickly within the fovea, so acuity is substantially reduced by fovea/parafovea boundary.) Therefore, the RVF locational gradient across the first three letters will be shallower than for LVF or CVF presentation. See Figure 6 for a comparison of the proposed activation patterns for CVF, RVF, and 
LVF presentation. Due to this shallowness, the second and third letter nodes already fire as early as possible, so additional top-down input from high $\mathrm{N}$ has no effect. (See Whitney and Lavidor (2005) for details.)

Figure 6 about here.

Therefore it should be possible to abolish the $\mathrm{N}$ effect (in the CVF and LVF) by increasing input to the internal letters (mimicking the top-down effect of high $\mathrm{N}$ ), and to create the $\mathrm{N}$ effect (in the RVF) by decreasing input to the internal letters (mimicking the steeper LVF and CVF gradients).

For CVF and LVF presentation, input to the internal letters can be increased by slightly dimming the first letter, thereby reducing feature-level, left-to-right inhibition from the first letter to the internal letters. For the LVF in particular, dimming the last letter may help to smooth the locational gradient, reproducing the moderating effect of a top-down activation gradient. See Figure 7. A small change in contrast level should have little direct effect on the perception of the letter. Therefore the dimming itself is not expected to increase RTs. For LVF and CVF presentation, such adjustments should mimic the compensatory effect of top-down input from high $\mathrm{N}$. Therefore, responses to low-N words should be facilitated (as compared to a low-N control condition), making low-N words as fast as high-N words, giving no $\mathrm{N}$ effect. The same manipulation in the RVF should have little effect.

\section{Figure 7 about here.}

Conversely, dimming the internal letters of an RVF string should mimic a steeper gradient, creating an $\mathrm{N}$ effect. That is, under such an adjustment, RTs for high-N words should be as fast as the control high-N condition (because the top-down input from high$\mathrm{N}$ compensates for the reduced bottom-up input), but RTs should increase in the low-N condition (because there is no such compensation).

In summary, for lateralized presentation, dimming the outer letters should mimic the effect of top-down input from high-N, abolishing the LVF $\mathrm{N}$ effect via facilitation of low-N words. Dimming the inner letters should reproduce the LVF activation pattern in the RVF, creating an $\mathrm{N}$ effect via inhibition of low-N words. For the CVF, dimming the first letter should reproduce the effect of high- $\mathrm{N}$, also abolishing the $\mathrm{N}$ effect via facilitation of low-N words. 
These predictions were confirmed in two lexical-decision experiments. In the first experiment, we focused on the asymmetry of the $\mathrm{N}$ effect, using lateralized presentation only. There were three presentation conditions: control, both outer letters dimmed, or both inner letters dimmed. In the control condition, all letters were presented in white on a dark gray background. A letter was dimmed by changing it to light gray. In the second experiment, we focused on negating the $\mathrm{N}$ effect for both the CVF and LVF. In this case, a single adjustment condition was tailored to specific locations. For lateralized presentation, both outer letters were dimmed; for the CVF, only the first letter was dimmed.

The results of the experiments are given in Figures 8 and 9. In both experiments, it is evident that dimming the outer letters had no effect in the RVF, but facilitated LVF low$\mathrm{N}$ words in particular, abolishing the $\mathrm{N}$ effect. Note that this facilitation could not be a result of simply unmasking the internal letters at a very low level, as this effect was not present for the RVF. Thus this manipulation had a VF-specific effect, as predicted. The second experiment shows that it is also possible to abolish the CVF $\mathrm{N}$ effect. The fact that low-N facilitation was achieved in the CVF indicates that the lack of facilitation in the RVF was not a result of more efficient processing in general.

Figures 8 and 9 about here.

The first experiment also shows that the $\mathrm{N}$ effect can be created in the RVF by dimming the internal letters, as predicted. These results firmly establish that the $\mathrm{N}$ effect depends on orthographic activation patterns. When top-down input can help the internal letters fire earlier, there is an $\mathrm{N}$ effect. When they already fire as early as possible relative to each other (due to a shallow acuity/locational gradient), there is no $\mathrm{N}$ effect.

Note also that across experiments, the majority (10 of 14) of the adjusted conditions did not differ from their respective controls, confirming the assumption that dimming would have little direct effect on the processing of the affected letters. Rather, differences from the control condition only occurred in those cases in which the adjustment was predicted to modulate the effect of low $\mathrm{N}$.

In summary, these experiments demonstrate that the asymmetries of the $\mathrm{N}$ and length effects do not arise from inherent differences in processing at the lexical level, because if that were the case, it would not be possible to reverse the asymmetries via visual 
manipulations. Rather, the $\mathrm{N}$ effect occurs when the slope of the locational gradient is sufficiently steep that top-down input can assist with the firing of the internal letters. The locational gradient is steeper for the LVF and CVF than for the RVF, explaining the pattern of the $\mathrm{N}$ effect. In contrast, the length effect occurs when the locational gradient is not smoothly decreasing. This only occurs for the LVF, explaining the pattern of the length effect.

This analysis suggests that it may be possible for top-down input from high-N to compensate for a non-smooth LVF gradient in longer words. Indeed, Lavidor and Ellis (2002b) showed that for high-N words of four to six letters, the LVF length effect was absent (but was present for low-N words). That is, the facilitatory effect of high $\mathrm{N}$ increased with length, canceling the length effect. These results show that performance under LVF presentation is very dependent on orthographic activation patterns, which can be adjusted via top-down input, or contrast manipulations. Indeed, Chiarello (1985) demonstrated that centrally presented orthographic primes produced facilitation for LVF, but not RVF, target presentation (under automatic priming conditions where the probability of a prime-target relationship was low). This LVF sensitivity may also contribute to asymmetries in semantic priming, as discussed next.

\section{Semantic Asymmetries}

Thus far, we have examined theoretical arguments and experimental evidence for a sub-lexical source of VF asymmetries associated with lexical processing. Next we consider how such a sub-lexical source could also lead to asymmetries in semantic processing. In the following, I will characterize the effect of LVF presentation as providing impaired orthographic encoding and reduced bottom-up input. This subsumes all effects of differences in letter activations that lead to a reduced match to connection weights and/or slowed activation of the target word.

Semantic Priming. Various attributes of the prime/target relationship can be manipulated under hemifield presentation the target. The prime can be presented to the $\mathrm{CVF}$, to the same hemifield as the target, or to the opposite hemifield. The target can be related to the prime by association, (e.g., SPIDER and WEB), shared semantic category (e.g., BEET and TURNIP), or by both attributes (e.g. NURSE and DOCTOR). The 
strength of a such semantic or associative relationship can also be varied. If the prime or target is ambiguous in meaning, the related word can refer to either the dominant meaning (e.g. BANK and MONEY) or to the subordinate meaning (e.g., BANK and RIVER). Manipulations of these factors have yielded different patterns of visual field asymmetries. First I will review the major findings. All studies discussed below are lexical-decision experiments.

Burgess and Simpson (1988) used central presentation of an ambiguous prime followed by presentation of a target related to the prime's subordinate or dominant meaning. Under a Stimulus Onset Asynchrony (SOA) of $750 \mathrm{~ms}$, priming of a dominant target occurred for both LVF and RVF presentation, while priming of the subordinate target occurred only for LVF presentation. In another experiment (Faust \& Lavidor, 2003), a pair of primes was centrally presented, and the targets were ambiguous. The primes were either both related to the target's dominant meaning, or both related to the subordinate meaning, or one was related to each meaning (mixed primes). For RVF targets, dominant primes were the most facilitatory of the three prime types; for LVF targets, mixed primes were the most facilitatory .

Chiarello, Burgess, Richards and Pollock (1990) compared priming of associativeonly, semantic-only, and associative-semantic relationships for primes and targets presented to the same hemifield under an SOA of $600 \mathrm{~ms}$. Associative-only primes produced no facilitation, and associative-semantic priming was present for both VFs, while semantic-only priming was present for LVF, but not RVF, presentation.

However, a subsequent study revealed the opposite pattern for semantic-only primes. Using prime presentation to the same or opposite visual field as the target under SOAs of 250 or $450 \mathrm{~ms}$, Abernathy and Coney (1996) found facilitation when the prime was presented to the RVF (independently of target location), while prime presentation to the LVF did not provide facilitation. Koviosto (1997), using SOAs of 165, 250, 500, and 750 $\mathrm{ms}$, showed that these conflicting results were related to the differing SOAs. For 165 and $250 \mathrm{~ms}$, facilitation was stronger for RVF primes, while for $750 \mathrm{~ms}$ facilitation was stronger for LVF primes, with a non-significant LVF advantage for $500 \mathrm{~ms}$.

Another study (Hutchinson, Whitman, Abeare \& Raiter, 2003) compared strongly associated pairs with more weakly associated pairs (where semantic similarity was not 
controlled). For $50 \mathrm{~ms} \mathrm{SOAs}$, priming was determined by the prime's presentation location - RVF primes gave facilitation for strong, but not weak, associates for target presentation to either VF, while LVF primes gave facilitation for both strong and weak associates. This pattern differs from semantic-only priming for brief SOAs, where priming was stronger for RVF primes. For $750 \mathrm{~ms}$ SOAs, LVF primes gave facilitation for strong associates only, although priming for weak associates just missed significance for the LVF/LVF prime/ target condition.

In understanding the observed asymmetries, there are two separate issues. When the prime is centrally presented, any VF differences must arise from differing influences of the prime on the activation of the target word. When the prime is laterally presented, VF effects can also arise from the way in which the primed word differentially interacts with other lexical and semantic representations in the lexicon.

Based on factors that we have already discussed, I address the resulting VF differences in both these cases. When the effect of the prime is held constant (central presentation), LVF target presentation can result in stronger priming because both semantic and orthographic priming are possible. That is, for RVF presentation, priming is limited to interactions between the semantic and lexical levels; for LVF presentation, interactions between the lexical and orthographic levels can lead to facilitation as well, resulting in greater total facilitation. We now consider how this proposal explains the experimental findings for ambiguous words.

I will assume that there are separate lexical representations for the different meanings of the word, and that there is a separate, semantic level of representation. Each lexical representation of the word inhibits unrelated meanings. The lexical representations of related words are primed via spreading activation within and between the semantic and lexical levels.

Figure 10 shows the proposed interactions for the results of Burgess and Simpson (1988). When the prime is presented, it activates both meanings at the lexical and semantic levels, as well as words associated with both meanings. Under a long SOA, the dominant meaning will inhibit the subordinate meaning, but words related to the subordinate meaning remain primed because they do not directly receive the inhibition related to the competition between the meanings. 
When a subordinate target is presented to the RVF, there is no facilitation, because it takes extra energy to co-activate the corresponding semantic representation (which has been inhibited by the dominant meaning.) Thus, although the lexical node is corresponding to the subordinate target is primed, this priming is not evident due to the interaction with the semantic level. For LVF presentation, the interaction between the semantic and lexical levels is the same as for RVF presentation. However, in this case, there is an opportunity for orthographic priming. The primed lexical node provides topdown input to the orthographic level, smoothing the locational gradient, and facilitating responses. This type of priming does not occur for the RVF because the locational gradient is already smoothly decreasing. Thus there is facilitation of the subordinate meaning for LVF, but not RVF, presentation.

Figure 10 about here.

Next we consider priming for an ambiguous target in Lavidor and Faust (2003). LVF priming should be strongest when top-down input to the orthographic level is maximized. This would occur when both of the target's lexical nodes are primed, namely in the mixed-primes condition, as observed. In contrast, RVF priming is strongest for dominant primes because priming is limited to the semantic/lexical levels, and dominant primes provide the strongest semantic priming.

What is the effect of lateralization of the prime? Because activation levels are lower under lateralized primes and so top-down influence from the lexical to orthographic levels is reduced, priming is dominated by the VF of the prime, not the target. We now consider the dynamics of lexical activation in more detail. When a prime is presented, the prime activates the corresponding lexical node, as well as exciting other semantic and lexical representations via spreading activation. However, lateral inhibition within the lexical level will serve to inhibit the same lexical items that have been primed. For example, for the prime DOCTOR, the DOCTOR word node is directly excited, while the NURSE node is excited via spreading activation. However the DOCTOR node also inhibits the NURSE node, due to the usual competition within the lexical level (and the NURSE node inhibits the DOCTOR node, as well). Thus related word nodes are both excited and inhibited. 
Now recall that LVF presentation results in impaired orthographic encoding and decreased bottom-up input to corresponding word. This would have two contradictory effects. The reduced bottom-up input to the prime's word node would provide less excitation to drive spreading activation to related words. However, the reduced input would also decrease the inhibitory capacity of the prime's word node. The net effect of reduced bottom-up input on the activation of a related word node then depends on the relative levels of decreased excitation versus decreased inhibition.

The net result of the decreased inhibition, in turn, will vary with the difference between the activation levels of the related word and the prime. As this difference increases, the prime is inhibited less by the related word, so it can inhibit the related word relatively more. For example, if two competing nodes are well matched (little difference in activation levels), it will take a long time for one node to dominate. If the initial activation level of the more highly activated node is held constant, but the initial activation level of the other node is decreased, the more highly activated node will dominate more quickly. Therefore, the competitive interaction can be approximated as a one-way inhibitory connection from the more excited node to the less excited node, where the connection weight increases with the difference in their initial activations. Decreased bottom-up input to the prime can be considered to reduce the effective connection weight between the prime and related words.

As a result of these dynamics, the activation of a related word is a non-linear function of prime activation. To illustrate, we consider a simple model in which the effect of spreading activation is given by a positive weight between the prime and related word node, and then the effect of competition is modeled via an inhibitory connection weight that is equal to the difference in activation between the prime and related word node. Let $\mathrm{P}$ denote the activation of the prime, and $\mathrm{E}$ denote the excitatory weight. The initial activation of the related word node, $\mathrm{R} 1$, is then given by $\mathrm{P}^{*} \mathrm{E}$. The inhibitory weight, $\mathrm{I}$, is equal to P - R1, and the resulting activation of the related word node, R2, is given by R1 $\mathrm{P}^{*} \mathrm{I}$. For $\mathrm{E}=0.5$, the corresponding values of $\mathrm{R} 2$ for $\mathrm{P}=0.2,0.6$, and 0.9 are given Table 1. As $\mathrm{P}$ increases from 0.2 to $0.6, \mathrm{R} 2$ increases from 0.08 to 0.12 . As $\mathrm{P}$ increases further (from 0.6 to 0.9 ), R2 decreases (from 0.12 to 0.045 ), as the effect of inhibition outweighs the effect of excitation. Thus the related word activation (R2) initially increases as a 
function of prime activation (for $\mathrm{P}=0.2$ to 0.6 ), but then decreases as a function of prime activation (for $\mathrm{P}=0.6$ to 0.9 ).

In summary, the effect of LVF presentation of the prime depends on how the reduced bottom-up input levels affect the balance of inhibition and excitation on related word nodes. Due to feedback interactions, the activation of a related word may be a non-linear function of the activation of the primed word node. As discussed next, this explains how VF asymmetries in priming effects could arise, and why VF asymmetries are dependent on SOAs and association strength.

First we consider semantic-only primes. I assume that as SOA increases, activation continues to accumulate for the primed word node. That is, prime activation is an increasing function of SOA. I also propose that for RVF prime presentation for SOAs $<$ $500 \mathrm{~ms}$, the activation of a related word node is an increasing function of prime activation. Because LVF prime presentation results in less bottom-up input to the prime (i.e., less prime activation), the activation of the related word node is higher for RVF primes than LVF primes, giving an RVF priming advantage. This would correspond to $\mathrm{P}$ $=0.2$ for $\mathrm{LVF}$ presentation and $\mathrm{P}=0.6$ for $\mathrm{RVF}$ presentation in the above example.

However, as SOA increases, RVF prime activations reach the level in which increased inhibition to the related word node outweighs increased excitation, and so the activation of the related word node decreases as prime activation increases. Thus for SOAs > $500 \mathrm{~ms}$, LVF primes result in higher related word activations than RVF primes, because the reduced LVF prime activations remain in the range where related word activations are an increasing function of prime activation. This would correspond to $\mathrm{P}=0.9$ for $\mathrm{RVF}$ presentation and $\mathrm{P}=0.6$ for LVF presentation in the above example. See Figure 11.

Figure 11 about here.

Why then is LVF priming stronger than RVF priming at short SOAs for weakly associated primes (Hutchinson, Whitman, Abeare \& Raiter, 2003)? It may be the case that a weakly associated prime provides less excitation to the target than a semantic-only prime. In this case, inhibition would have a relatively stronger effect, and increased inhibition may outweigh the increased excitation even at short SOAs. For example, consider $\mathrm{E}=0.4$ in the above example, corresponding to weaker excitatory effect. In this case, $\mathrm{P}=0.2$ gives $\mathrm{R} 2=0.056$, while $\mathrm{P}=0.6$ gives $\mathrm{R} 2=0.024$. So for $\mathrm{E}=0.4, \mathrm{R} 2$ is a 
decreasing function of $\mathrm{P}$ in the range 0.2 to 0.6 , while for $\mathrm{E}=0.5$, $\mathrm{R} 2$ was increasing function of $\mathrm{P}$ in this range. So it may be the case that a weakly associated prime corresponds to the lower value of $\mathrm{E}$, where related node activations are decreasing function of $\mathrm{P}$ even for low values of $\mathrm{P}$. Therefore an LVF prime advantage arises even at short SOAs. As SOA increases, priming should decrease because the increased values of $\mathrm{P}$ lead to relatively stronger inhibition. Indeed, for $750 \mathrm{~ms}$ SOA, weak associative priming was absent or nearly absent for LVF primes (Hutchinson, Whitman, Abeare \& Raiter, 2003).

While these explanations of the various priming results may seem somewhat complex, they are based on the basic idea of a single mode of lexical access to a single lexicon. Under any model of visual word recognition, there would have to be a delicate balance between excitatory and inhibitory forces within and between processing levels. I claim that such complex, non-linear interactions, modulated by the optimality of orthographic encoding, are sufficient to explain any VF differences. Even if the present explanations of the observed asymmetries are not precisely correct, they demonstrate in principle how seemingly semantic asymmetries could arise from differences in orthographic encoding. In contrast, the usual explanation of semantic-priming asymmetries relies on separate modes of lexical access to separate lexicons. These more complicated assumptions are unnecessary, and are inconsistent with brain-imaging data.

Shears and Chiarello (2003) characterize the semantic priming data as indicating that RVF presentation results in the rapid and narrow activation of related meanings, while LVF presentation results in a slower and broader activation of related meanings. This summary can be understood in a way that is consistent with a single route to the lexicon. LVF activation of lexical items (and related meanings) is slower due to an impaired orthographic encoding. LVF activation is broader because the reduced activation of the corresponding word decreases its inhibitory capacity, allowing related lexical items to remain activated.

This analysis suggests that it may be more difficult to use a contrast adjustment to get the LVF to look like the RVF for semantic asymmetries than in the length or $\mathrm{N}$ experiments. In those experiments, it was sufficient to change bottom-up input levels relative to $L V F$ conditions in order to create the usual RVF RT pattern in the LVF. To 
abolish the length effect, the LVF six-letter RT had to be reduced to that of the LVF fourletter RT. To abolish the N effect, the LVF low-N RT had to be reduced to that of the LVF high-N RT. However, in semantic priming, the LVF contrast adjustment may need to mimic the input level of the $R V F$ control condition in order to reproduce the RVF priming pattern. That is, the semantic priming pattern may depend on the absolute level of bottom-up input. Thus, if the LVF contrast adjustment is not sufficient to fully compensate for LVF presentation (i.e., if it does reduce the LVF RT all the way to the RVF control level), the RVF semantic-priming pattern may not be fully replicated. Conversely, if RVF bottom-up input levels are not degraded all the way to LVF levels, the LVF pattern may not appear in the RVF.

Indeed, our preliminary data from the application of contrast adjustments to the Faust and Lavidor (2003) task indicate that the adjustments give a priming pattern in both VFs that is intermediate between the control LVF and RVF patterns. That is, the manipulations seem to abolish this semantic asymmetry, rather than reverse it.

Asymmetry of the N400. Kutas and Federmeier (1999) have demonstrated an asymmetry that occurs with respect to the N400 effect in sentence processing. It is well known that the amplitude of the N400 is modulated by how well a word fits into the context of a sentence (Kutas \& Hillyard, 1980). In general, expected words give lower (less negative) N400 amplitudes than unexpected words. Therefore, N400 amplitude has been taken to index the ease with which the meaning of a word can be integrated into the semantic representation of a sentence.

Federmeier and Kutas (1999) investigated this effect for three conditions: a) an expected word; b) an unexpected word within the same semantic category as the expected word (within-category violation); and c) an unexpected word in a different, but related, semantic category (between-category violation). For example:

They wanted to make the hotel look more like a tropical resort, so along the driveway they planted rows of: a) palms b) pines c) tulips.

Here pines and tulips are both unexpected, but pines are trees, like the expected word palms, while tulips are not.

For central presentation of all words in the sentence (including the critical word), they found that semantic similarity to the expected word modulated the N400: within-category 
violations gave smaller amplitudes than between-category violations (with expected words giving the smallest amplitudes). For lateralized presentation of the critical word (with central presentation of the sentential context), the same pattern arose for RVF presentation. For LVF presentation, the N400 amplitudes of expected words and between-category violations were the same as the RVF amplitudes, respectively, but the amplitude of within-category violations increased, making it equal to that of the betweencategory violation. Thus, in the LVF, there was no effect of the degree of similarity to the expected word. The authors interpreted this asymmetry as indicating that the two hemispheres specialize in different aspects of semantic integration, as quoted in the Introduction (Kutas \& Federmeier, 1999).

Before explaining this asymmetry, we must first understand what the N400 is measuring. The Federmeier and Kutas (1999) result for central presentation indicates that the N400 is primarily sensitive not to how well a word fits into the sentence, but rather to the degree of semantic priming. That is, the semantic features activated by the expected word also pre-activate related words to varying degrees, and the N400 indexes this priming. For example, pines shares more features with palms than does tulips, so pines is more highly primed than tulips, which is reflected in a reduced N400 component. This result is entirely consistent with the N400 effect in non-sentential contexts. For word lists, N400 amplitude decreases with a word's semantic similarity to previous words (Bentin, Kutas \& Hillyard, 1993).

However, N400 amplitude is also modulated by factors unrelated to semantic priming. For word lists and lexical decision, N400 amplitude is larger for low frequency words than high frequency words (Rugg \& Doyle, 1992; Debruille, 1998). For words and non-words in lexical decision, N400 amplitude increases with orthographic neighborhood size $(\mathrm{N})$, while N400 amplitude is higher overall for non-words than words. For lowfrequency words in lexical decision, N400 amplitude is larger for words that resemble a higher-frequency word than for ones that do not (e.g. BRIBE vs. CIRCUS; BRIBE is similar to BRIDE while CIRCUS is not similar to any higher-frequency word) (Debruille, 1998). In a task where two stimuli (S1, S2) were successively shown, and the subject had to specify whether or not a probe stimulus was the same as S1 and/or S2, there was N400 priming of S2 even when it was pseudoword that did not resemble a real word (a non- 
derivational pseudoword). That is, when S1 and S2 were the same non-derivational pseudoword, an N400 component was present for S1, but was completely absent for S2 (Deacon, Dynowska, Ritter \& Grose-Fifer, 2004).

Together, these results suggest that the N400 measures total lexical activation over the course of the activation of the target word, which depends on how efficiently the target word can inhibit its competitors. When a word is highly primed, it gets a head start on inhibiting competitors; total lexical activation and N400 amplitude are reduced as compared to unprimed targets. When a word has more neighbors, N400 amplitude increases because there are more highly activated competitors. A low-frequency word is less efficiently activated than a high-frequency word, so it inhibits competitors less efficiently, resulting in an increased N400 amplitude. When a low-frequency word is orthographically similar to a higher-frequency word, N400 amplitude is increased by the activation of the similar word. For non-words in lexical decision, total lexical activation and N400 amplitude are the highest, because there is no matching target to inhibit similar words. However, for repeated pseudowords, the N400 component is absent because lexical access is not even attempted, as the subject already knows that it is not a word (Deacon et al., 2004). In sum, I propose that the N400 component does not reflect semantic processing per se, but rather reflects lexical processing, where N400 amplitude is inversely related to how efficiently activation can be narrowed to a single word (the target).

Now we consider the source of the N400 asymmetry. As discussed above, LVF presentation would result in less bottom-up activation to the corresponding (target) word node. The amount of this decrease to the target would be larger than the decrease to word nodes that are orthographically similar to the target (i.e., the target has the most to lose). As a result, activation is less focused on the target. This would reduce the efficacy of inhibition from the target to orthographic competitors, and increase N400 amplitude. If so, why did LVF presentation only affect the within-category N400 (relative to RVF presentation)? As shown in Figure 12, such a pattern could arise if N400 amplitude is a non-linear, sigmoid function of inhibition efficacy. The between-category and expected word conditions give ceiling and floor effects, respectively, while the between-category condition falls in the more sensitive range. Furthermore, the level of pre-activation of the 
highly expected word is independent of presentation location, so reduced input to an unexpected word would also affect its ability to inhibit the expected word. Therefore, the increased LVF N400 for the within-category violation may also reflect a reduced ability to compete with the expected word. (Again, the lack of a VF effect for the betweencategory violation may reflect a ceiling effect.)

Figure 12 about here.

Thus I propose that the lack of an LVF similarity effect results from a decreased advantage for the target over competitors, arising from non-optimal LVF visual encoding. However, Kutas and Federmeier (2002) have also shown a similar pattern of asymmetry when the target was presented as a picture, rather than a word (i.e., an N400 difference for violation conditions in the RVF, but not the LVF). They took this result as showing that the N400 asymmetry is purely semantic in nature, contrary to the claim I'm making here. However, in the picture experiment, the lack a similarity effect in LVF arose because the between-category condition varied with VF. That is, the between-category amplitude decreased for the LVF, bringing it down to the level of the within-category violation. In contrast, in the word experiment, VF affected N400 amplitude for the within-category condition. This indicates that the lack of an LVF similarity effect in the picture experiment arose for a different underlying reason than in the word experiment. Therefore, the picture experiment by no means disproves the account offered here.

\section{Discussion}

The implications of the brain-imaging evidence, the length and $\mathrm{N}$ experiments, and the present discussion of semantic asymmetries are quite straightforward. Visual halffield studies are inappropriate for making claims about hemispheric specializations at the lexical and/or semantic levels. Brain-imaging indicates a single lexical route to the lexicon (Cohen et al., 2000; Dehaene et al., 2004; Tarkiainen, et al. 1999), as would be expected on the basis of parsimony. That is, it would be inefficient to have separate modes of lexical processing for the LVF and RVF, and it is unclear how separate modes could be integrated together for a string crossing both VFs. Moreover, theoretical and experimental investigations based on the SERIOL model show that VF asymmetries can and do arise from orthographic activation patterns (Whitney, 2004; Whitney \& Lavidor, 
2004; Whitney \& Lavidor, 2005). Therefore, it should be assumed that asymmetries in half-field studies reflect differences originating in pre-lexical, visual processing.

This is not to say that the usual strong RVF advantage for lexical processing is unrelated to the LH specialization for language. Consistent with the evidence for lateralization to the $\mathrm{LH}, \mathrm{I}$ assume that orthographic information falling into the LVF/RH is transferred across the corpus callosum, activating LH representations encoding LVF retinal locations. Such callosal transfer could well affect the resulting LH activation pattern, changing absolute and relative activation levels across that portion of the string. For example, callosal transfer may further degrade the non-optimal LVF/RH locational gradient for a left-to-right language. In a right-to-left language, the acuity gradient would be inverted for the RVF/LH, not the LVF/RH. In this case, the effect of acuity-gradient inversion may be reduced (relative to a left-to-right language) because the activation pattern does not also undergo callosal transfer, while the effect of callosal transfer alone on activation patterns (in the absence of acuity-gradient inversion in the LVF/RH) may create an LVF disadvantage (Lavidor \& Whitney, 2005). The important point is that these influences originate pre-lexically. At lexical access and beyond, there are no qualitative differences in processing arising from presentation location or reading direction. Rather, the quantitative effects of callosal transfer and acuity-gradient inversion have repercussions at these higher levels of processing.

In closing, I have two suggestions regarding future experimental work into visual word recognition. First, the role of behavioral studies should not be neglected. While brain imaging studies can localize where and when various stages of processing occur, they usually do not reveal how those brain areas carry out the processing being investigated. I believe that behavioral studies are actually more suitable for revealing the how. Due to the relative ease of running such studies, many different experimental conditions can easily be tested. In-depth analysis of the behavioral patterns can then inform us as to what algorithms the brain is using to perform a task. For example, the SERIOL model was developed primarily via consideration of priming, RT, and error patterns for letter and word recognition experiments. The proposed processing levels in the model fit well with brain imaging data that have since become available. 
That said, I would also suggest that more emphasis be placed on performing halffield experiments in brain-imaging studies. The high spatial and temporal resolution provided by modern techniques may further clarify the point of callosal transfer of orthographic information, as well as the effects of visual manipulations. Such knowledge would help us to better understand how the brain carries out the task of visual word recognition. 


\section{References}

Abernathy, M. \& Coney, J. (1996) Semantic category priming in the left hemisphere. Neuropsychologia, 34, 339-350.

Andrews, S. (1997) The effect of orthographic similarity on lexical retrieval: Resolving neighborhood conflicts. Psychonomic Bulletin and Review, 4, 439-461.

Bentin, S., Kutas, M. \& Hillyard, S.A. (1993) Electrophysiological evidence for task effects on semantic priming in auditory word processing. Psychophysiology, 30, 161-169.

Bouma, H. (1973) Visual interference in the parafoveal recognition of initial and final letters of words. Vision Research, 13, 767-782.

Burgess, C. \& Simpson, G. B. (1988) Cerebral hemispheric mechanisms in the retrieval of ambiguous word meanings. Brain and Language, 33, 86-103.

Chiarello, C. (1985) Hemisphere dynamics in lexical access: automatic and controlled priming. Brain and Language, 46, 146-172.

Chiarello, C., Burgess, C., Richards, L. \& Pollock, A. (1990) Semantic and associative priming in the cerebral hemispheres: some words do, some words don't ... sometimes, some places. Brain and Language, 38, 75-104.

Coltheart, M., Davelaar, E., Jonasson, J.T., \& Besner, D. (1977) Access to the internal lexicon. In S. Dornic (Ed.) Attention and Performance VI: The Psychology of Reading. London: Academic Press. 
Cohen L., Dehaene S., Naccache L., Lehericy S., Dehaene-Lambertz G., Henaff M.A., \& Michel F (2000) The visual word form area: Spatial and temporal characterization of an initial stage of reading in normal subjects and posterior split-brain patients. Brain, 291307.

Cohen, L., Martinaud, O., Lemer, C., Lehericy, S., Samson, Y., Obadia, M., Slachevsky, A. \& Dehaene, S. (2003) Visual word recognition in the left and right hemispheres: anatomical and functional correlates of peripheral alexias. Cerebral Cortex, 13, 13131333.

Deacon, D., Dynowska, A., Ritter, W. \& Grose-Fifer, J. (2004) Repetition and semantic priming of nonwords: Implications for theories of $\mathrm{N} 400$ and word recognition. Psychophysiology, 41, 60-74.

Debruille, J. B. (1998) Knowledge inhibition and N400: a study with words that look like common words. Brain and Language, 62, 202-220.

Dehaene, S., Jobert, A., Naccache, L., Ciuciu, P., Poline, J.B., Le Bihan, D., \& Cohen L. (2004) Letter binding and invariant recognition of masked words: behavioral and neuroimaging evidence. Psychological Science, 15, 307-313.

Ellis, A. W., Young, A.W. \& Anderson, C. (1988) Modes of word recognition in the left and right cerebral hemispheres. Brain and Language, 35, 254-273.

Estes, W. K., Allemeyer, D. H. \& Reder, S. M. (1976) Serial position functions for letter identification at brief and extended exposure durations. Perception \& Psychophysics, 19, $1-15$.

Faust, M. \& Lavidor, M. (2003) Semantically convergent and semantically divergent priming in the cerebral hemispheres: lexical decision and semantic judgment. Cognitive Brain Research, 17, 585-597. 
Federmeier, K. \& Kutas, M. (1999) A rose by any other name: long-term memory structure and semantic processing. Journal of Memory and Language, 41, 465-495.

Grainger, J. \& Whitney, C. (2004) Does the huamn mnid raed wrods as a wlohe? Trends in Cognitive Sciences, 8, 58-59.

Holcomb, P.J., Grainger, J., \& O'Rourke, T. (2002). An electrophysiological study of the effects of orthographic neighborhood size on printed word perception. Journal of Cognitive Neuroscience, 14, 938-950.

Hopfield, J.J. (1995) Pattern recognition computation using action potential timing for stimulus representation. Nature, 376, 33-36.

Hutchinson, A., Whitman, R.D., Abeare, C. \& Raiter J. (2003) The unification of mind: Integration of hemispheric semantic processing. Brain and Language, 87, 361-368.

Koivisto, M. (1997) Time course of semantic activation in the cerebral hemispheres. Neuropsychologia, 35, 497-504.

Koivisto, M., \& Laine. M. (2000) Hemispheric asymmetries in activation and integration of categorical information. Laterality, 5, 1-21.

Kutas, M. \& Federmeier, K. D. (1999) Right words and left words: electrophysiological evidence for hemispheric differences in meaning processing. Cognitive Brain Research, 8, 373-392.

Kutas, M. \& Federmeier, K. D. (2002) Picture the difference: electrophysiological investigations of picture processing in the two cerebral hemispheres. Neuropsychologia, 40, 730-747. 
Kutas, M. \& Hillyard, S. A. (1980) Reading senseless sentences: brain potentials reflect semantic incongruity. Science, 207, 203-205.

Lavidor, M \& Ellis, A. (2002a) Orthographic neighborhood effects in the right but not in the left cerebral hemisphere. Brain and Language, 80, 63-76.

Lavidor, M \& Ellis, A. (2002b) Word length and orthographic neighborhood size effects in the left and right cerebral hemispheres. Brain and Language, 80, 45-62.

Lavidor, M. \& Walsh, V. (2003). A magnetic stimulation examination of orthographic neighborhood effects in visual word recognition. Journal of Cognitive Neuroscience, 15, 354-363.

Lavidor, M. \& Whitney, C. (2005) Word length effects in Hebrew. Cognitive Brain Research, in press.

Lisman, J.E. \& Idiart, M.A.P. (1995) Storage of $7+-2$ short-term memories in oscillatory subcycles. Science, 267, 1512-1515.

McCandliss, B.D., Cohen, L. \& Dehaene, S (2003). The visual word form area: Expertise for reading in the fusiform gyrus. Trends in Cognitive Science, 7, 293-299 .

Rugg, M.D. \& Doyle, M.C. (1992) Event-related potentials and recognition memory for low- and high-frequency words. Journal of Cognitive Neuroscience, 4, 69-79.

Shears, C. and Chiarello, C. (2003) No go on neutrals? An interhemispheric account of semantic category priming. Laterality, 8, 1-23.

Tarkiainen, A., Helenius, P., Hansen, P.C., Cornelissen, P.L. \& Salmelin R. (1999) Dynamics of letter string perception in the human occipitotemporal cortex. Brain, 122, 2119-2132. 
Westheimer, G (1987) Visual Acuity. In R.A. Moses and W.M Hart (Eds) Adler's Physiology of the Eye, Clinical Applications (Chapter 17) St. Louis: The C.V. Mosby Company.

Whitney, C. (2001a) How the brain encodes the order of letters in a printed word: The SERIOL model and selective literature review. Psychonomic Bulletin and Review, 8, 221-243.

Whitney, C. (2001b) Position-specific effects within the SERIOL framework of letterposition coding. Connection Science, 13, 235-255.

Whitney, C. (2002) An explanation of the length effect for rotated words. Cognitive Systems Research, 3, 113-119.

Whitney, C. (2004) Hemisphere-specific effects in word recognition do not require hemisphere-specific modes of access. Brain and Language, 88, 279-293.

Whitney, C., \& Berndt, R.S. (1999) A new model of letter string encoding: Simulating right neglect dyslexia. Progress in Brain Research, 121, 143-163.

Whitney, C. \& Lavidor, M. (2004) Why word length only matters in the left visual field. Neuropsychologia, 42, 1680-1688.

Whitney, C. \& Lavidor, M. (2005) Facilitatory orthographic neighborhood effects: The SERIOL model account. Submitted.

Wolford, G. \& Hollingsworth S. (1974) Retinal location and string position as important variables in visual information processing. Perception \& Psychophysics, 16, 437-442. 
Young, A. W. \& Ellis A.W. (1985) Different methods of lexical access for words presented to the left and right visual hemifields. Brain and Language, 24, 326-358. 


\begin{tabular}{|l|l|l|l|}
\hline $\mathbf{P}$ & $\mathbf{E} * \mathbf{P}=\mathbf{R} 1$ & $\mathbf{P}-\mathbf{R} 1=\mathbf{I}$ & $\mathbf{R} 1-\mathbf{I} * \mathbf{P}=\mathbf{R 2}$ \\
\hline 0.2 & 0.1 & 0.1 & $0.10-0.02=0.08$ \\
\hline 0.6 & 0.3 & 0.3 & $0.30-0.18=0.12$ \\
\hline 0.9 & 0.45 & 0.45 & $0.45-0.405=0.045$ \\
\hline
\end{tabular}

Table 1: Values of R2 (related word node activation) for various values of P (prime node activation) for $\mathrm{E}=0.5$. 


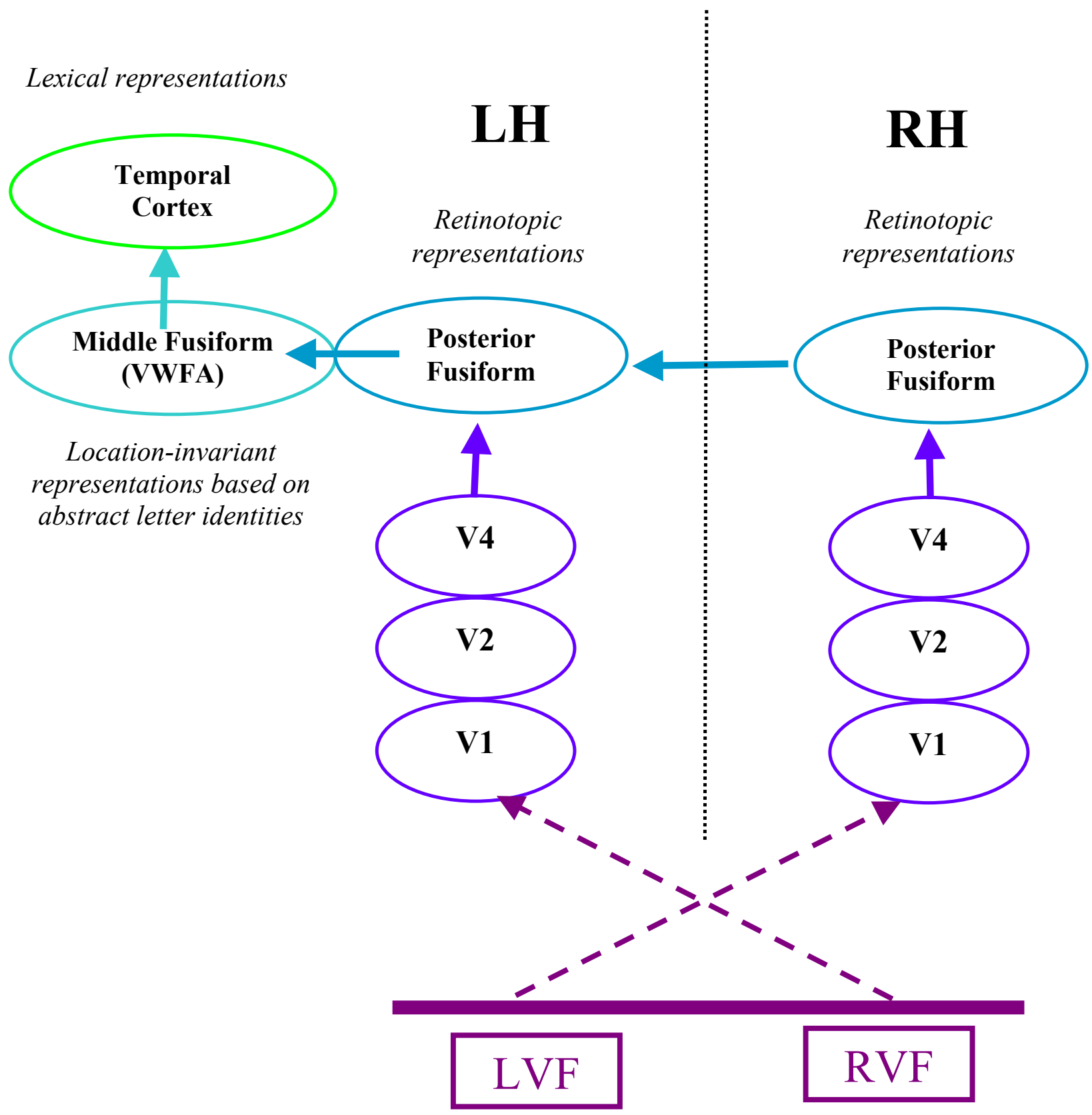

Figure 1: Emerging picture of visual processing of letter strings in normal readers, based on brain-imaging data (Cohen et al., 2000; Dehaene et al., 2004; Tarkiainen, et al. 1999). For both LVF and RVF presentation, information is funneled through LH fusiform areas, which encode a pre-lexical representation of the string. Any effect of VF would have to originate prior to this LH convergence. 


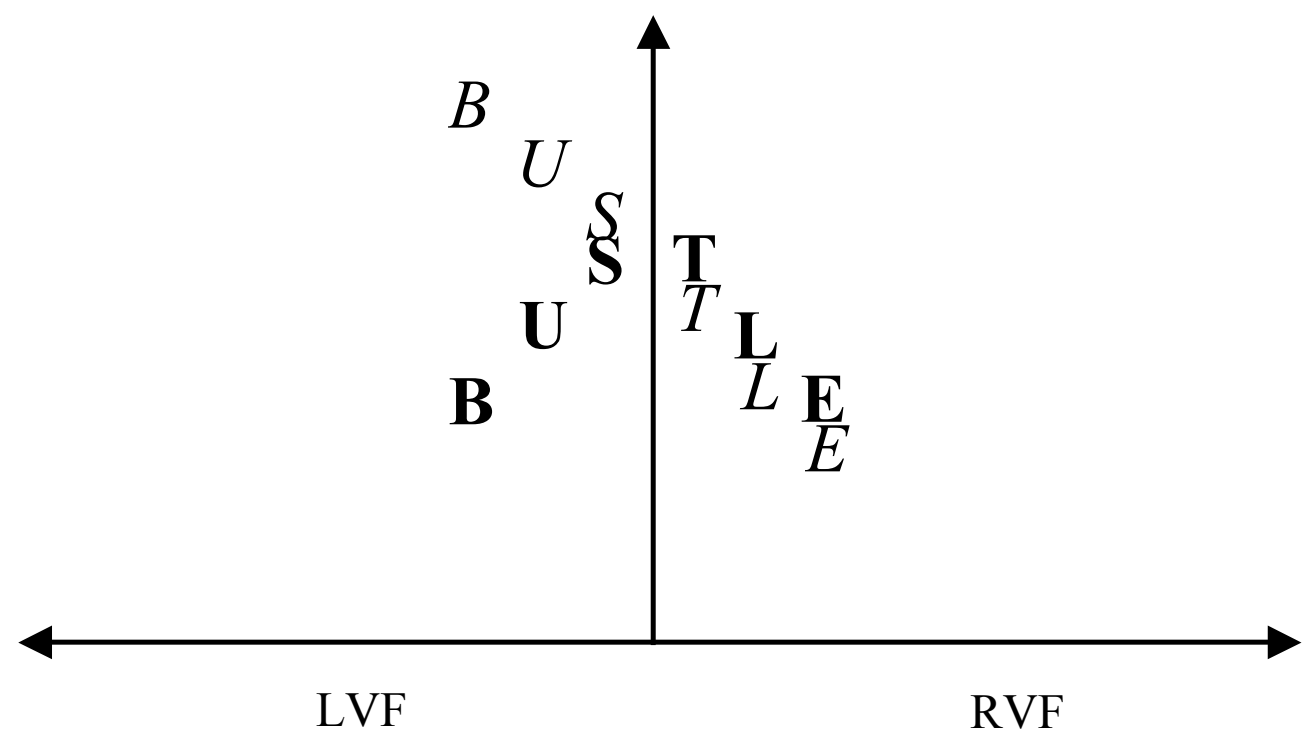

Figure 2: Acuity gradient (boldface) versus locational gradient (italics) for the fixated stimulus BUSTLE. The horizontal axis represents retinal location, while the vertical axis represents activation level. 
Activation Level

a)

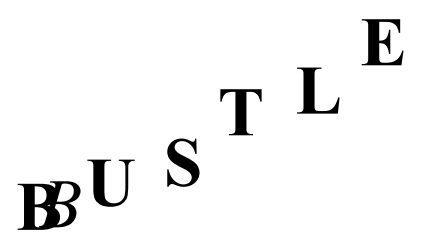

$U$

$S$

$T L E$

BB

\section{${ }^{\mathbf{U}} \mathbf{S S}_{T_{T}}{ }_{\mathbf{L} L E}$}

LVF/RH

RVF/LH

Figure 3: Activation patterns for parafoveal presentation. Boldface letters represent bottom-up excitation to the feature level, illustrating stronger excitation to the LVF/RH. Lateral inhibition then forms the locational gradient (italics). In the LVF/RH, strong inhibition from the first letter has a large effect on the second and third letters; however, due the relatively low activations of the initial letters and the increasing activation levels of the final letters, lateral inhibition fails to create a smoothly decreasing gradient near the end of the string. In the $\mathrm{RVF} / \mathrm{LH}$, the locational gradient is equivalent to the bottomup acuity gradient, so it remains smoothly decreasing. 


\section{Activation Level}

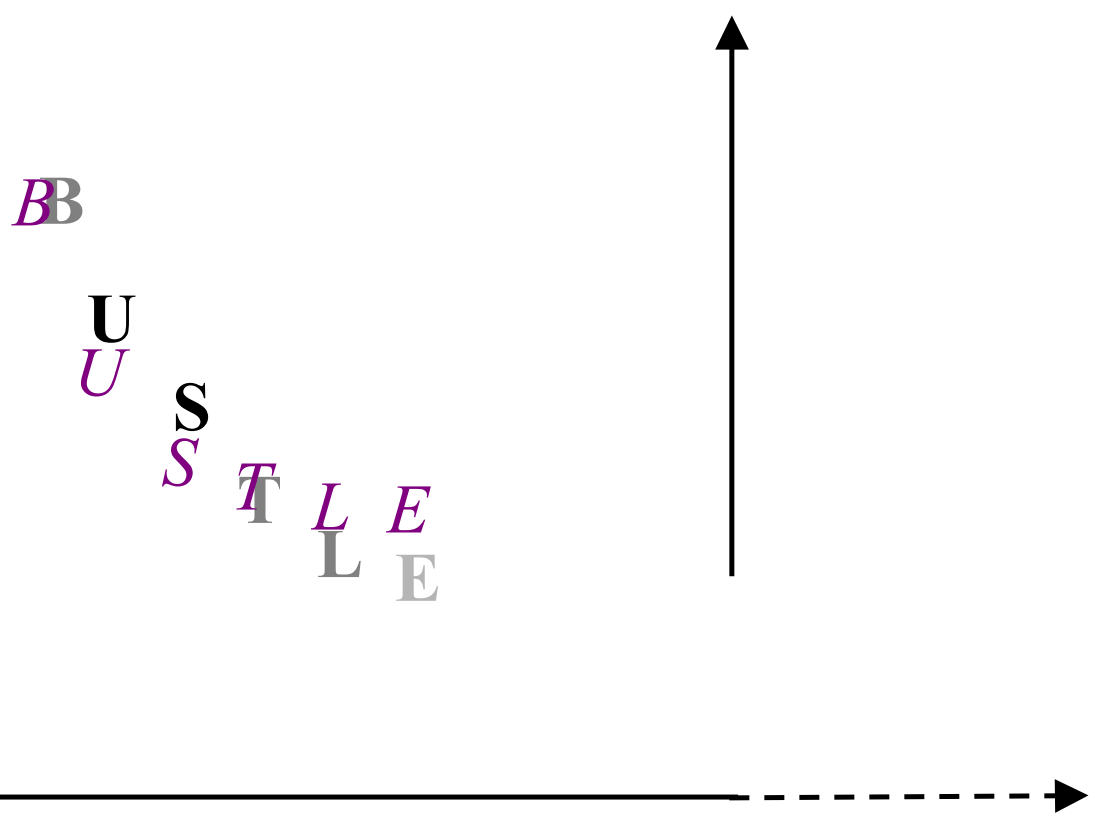

LVF/RH

Figure 4: LVF/RH locational gradient (purple italics) and corrective effect (normal face) the contrast manipulation. Increased contrast is indicated in black (second and third letters), decreased contrast is shown in light gray (sixth letter), while unchanged contrast is shown in medium gray. The increased bottom-up input to the second and third letters raises their activation levels, and increases left-to-right lateral inhibition to the fourth, fifth and sixth letters, decreasing their activation levels. Decreased bottom-up input to the sixth letter further lowers its activation level. The result is a more smoothly decreasing activation gradient. 


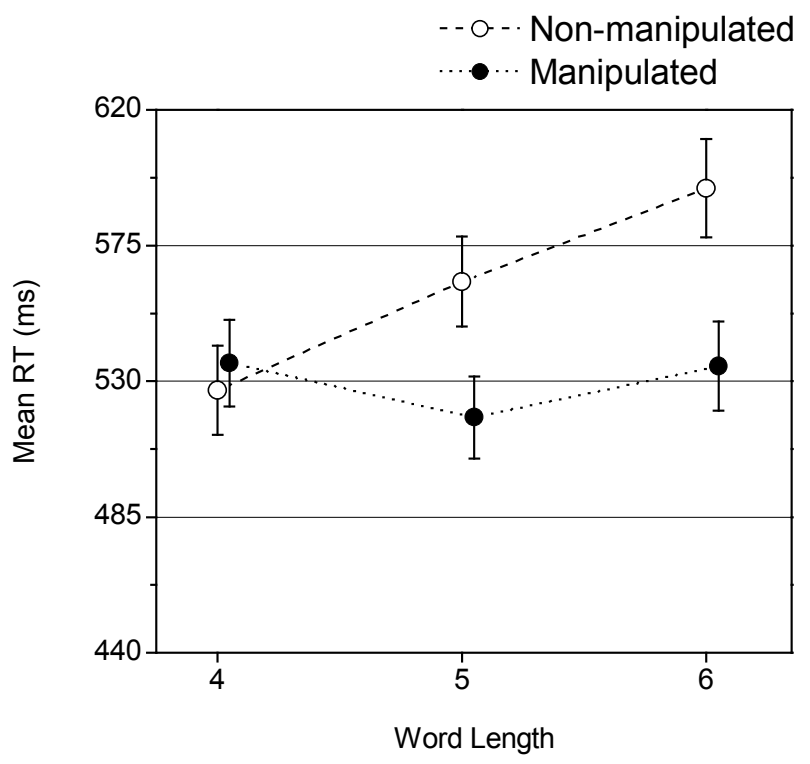

LVF

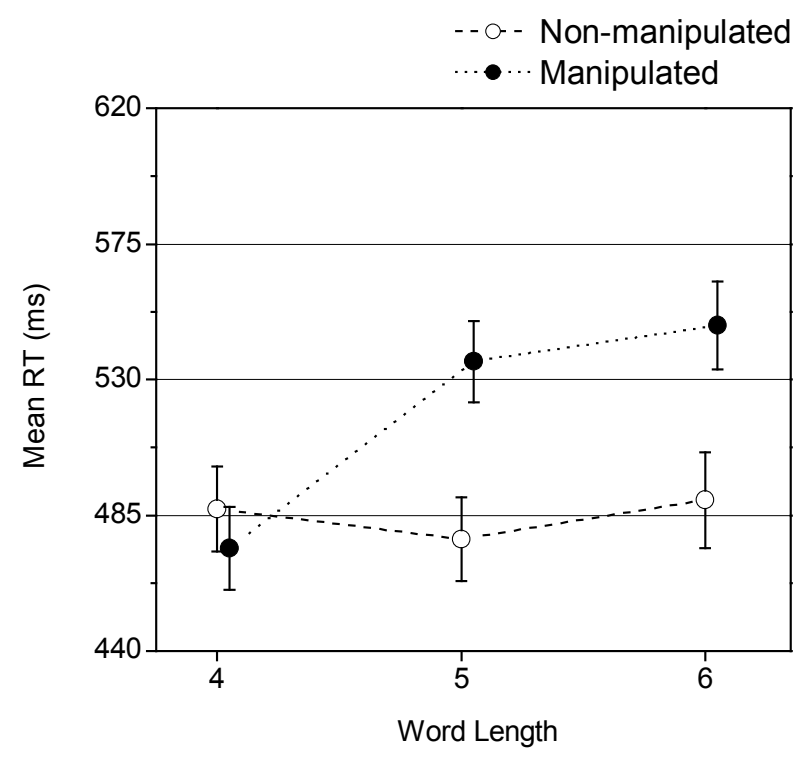

RVF

Figure 5: Experimental results of lexical-decision experiment from Whitney and Lavidor (2004). In the non-manipulated condition, all letters were presented at the same contrast level. In the manipulated condition, the contrast of the second and third letters was increased, and the contrast of the six letter (if present) was decreased. Error bars represent one standard error of the mean. 


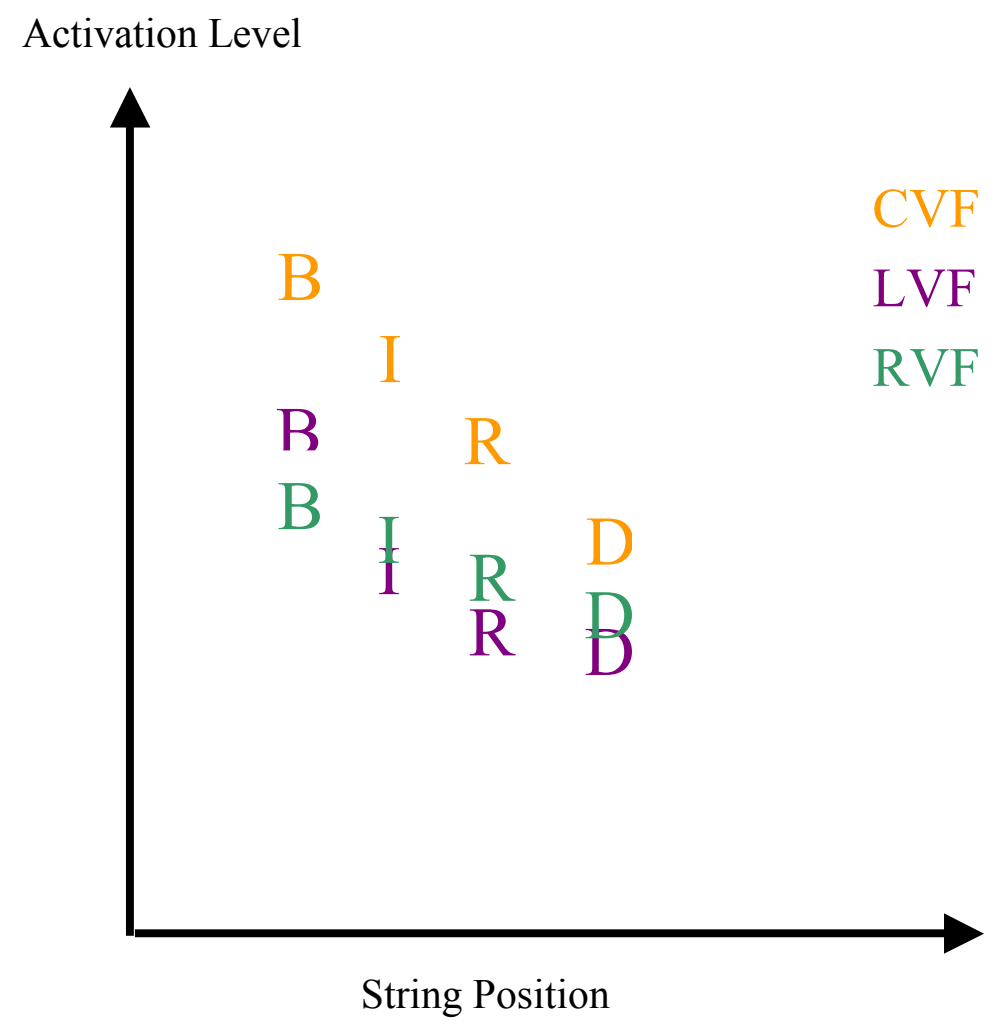

Figure 6: Comparison of proposed feature-layer activation patterns (locational gradients) for CVF, LVF, and RVF presentation of the stimulus BIRD. The CVF pattern is steeply and smoothly decreasing. The LVF pattern is non-smooth; it is steep across positions 1 to 3 , and shallow across positions 3 to 4 , because left-to-right inhibition becomes increasingly less effective as acuity increases. The RVF pattern is smooth and shallow, because it follows the acuity gradient, which is shallow in the parafovea. 


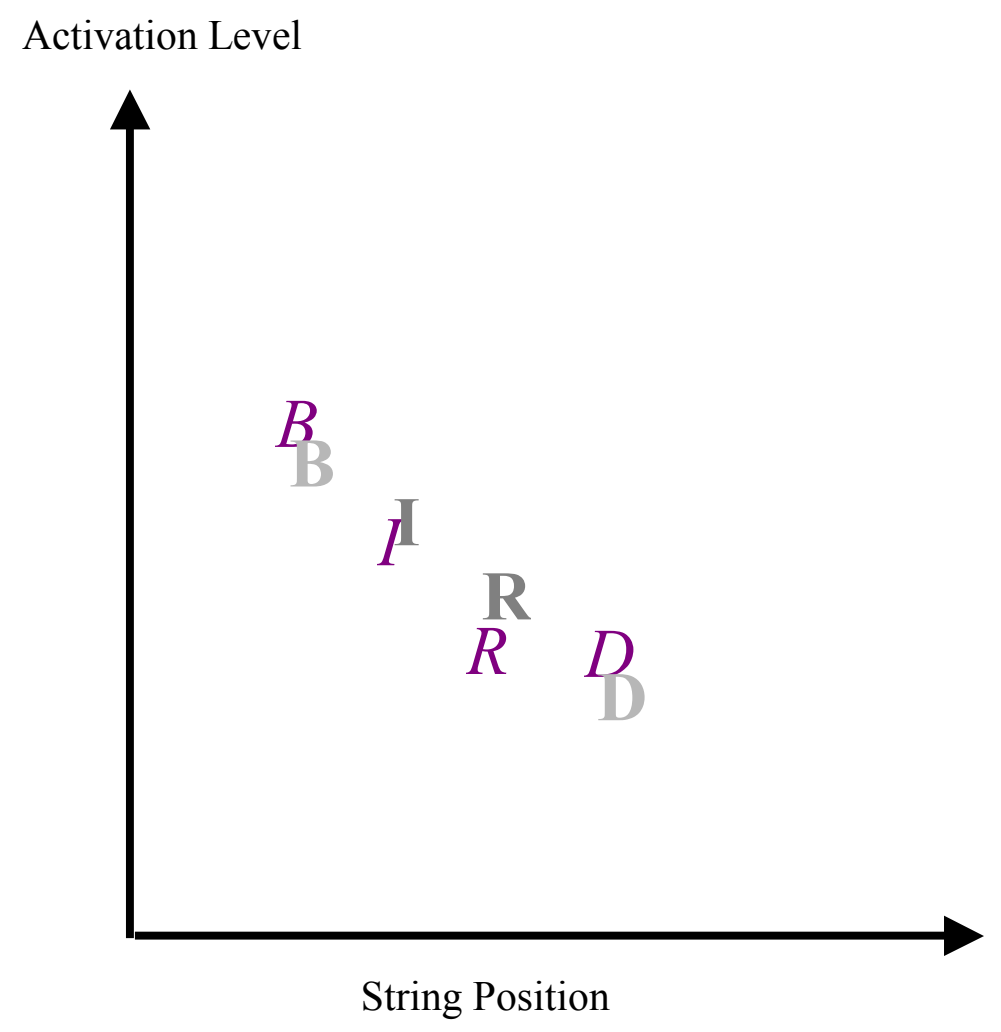

Figure 7: $\mathrm{LVF} / \mathrm{RH}$ locational gradient (purple italics) and effect (normal face) reducing the contrast of the first and fourth letters (indicated in light gray). The decreased bottomup input to the first letter decreases its activation level. The decreased activation of the first letter decreases left-to-right lateral inhibition to the other letters, increasing their activations, while the decreased bottom-up input to fourth letter has the net effect of decreasing its activation. The result is a smoother, shallower locational gradient. 


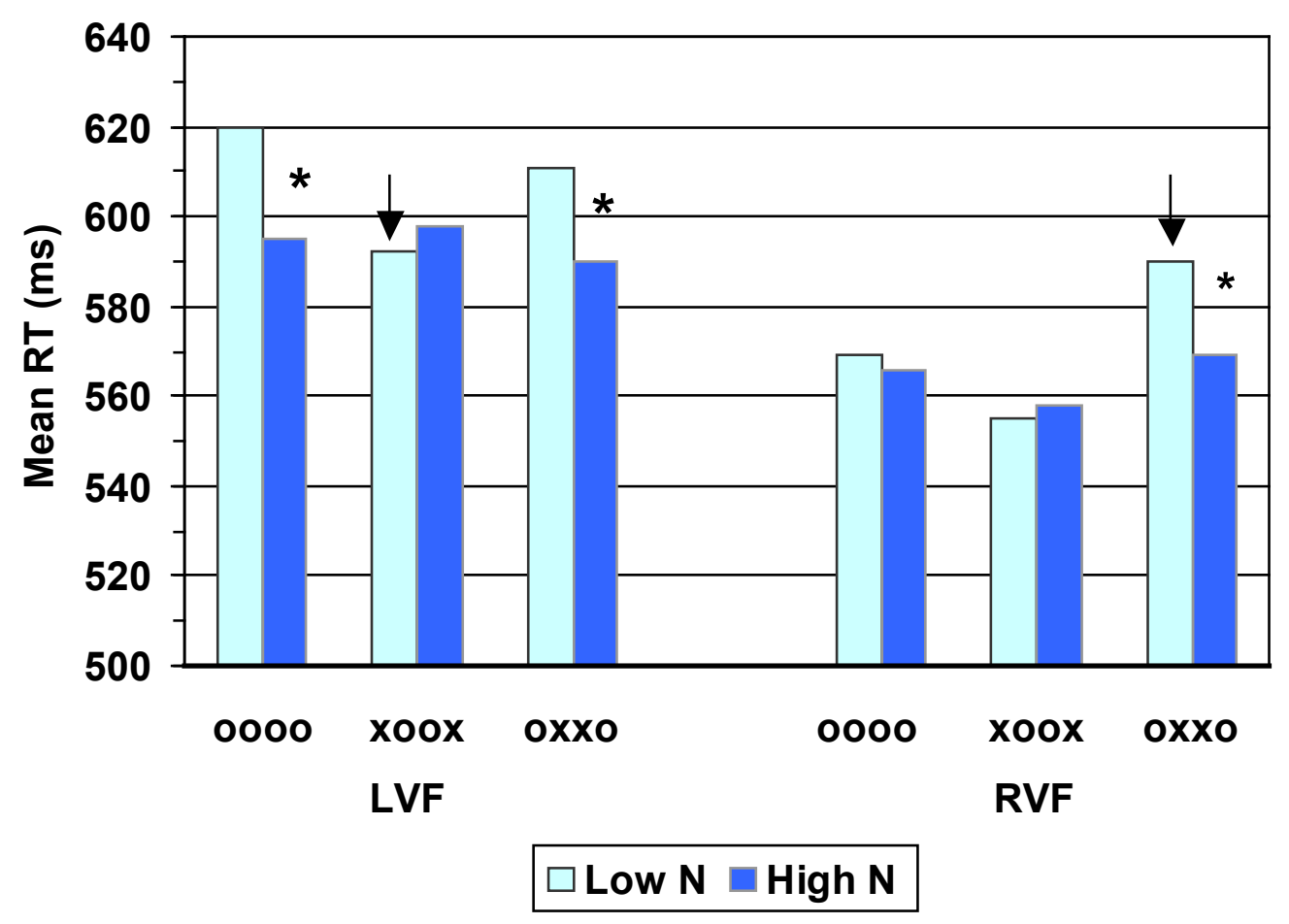

Figure 8: Experimental results for contrast adjustments for lateralized presentation of high- $\mathrm{N}$ versus low-N words in lexical decision. The presentation condition is indicated by o's and $\mathbf{x}^{\text {‘s}}$, which represent control and dimmed contrast levels, respectively, for the letter in the corresponding position. For example, oooo denotes the control condition, while xoox represents the condition in which the outer letters were dimmed. A statistically significant $(\mathrm{p}<0.5)$ difference between high-N and low-N conditions is indicated with a star. A contrast-adjusted condition that significantly differed from its respective control presentation is indicated by an arrow. 


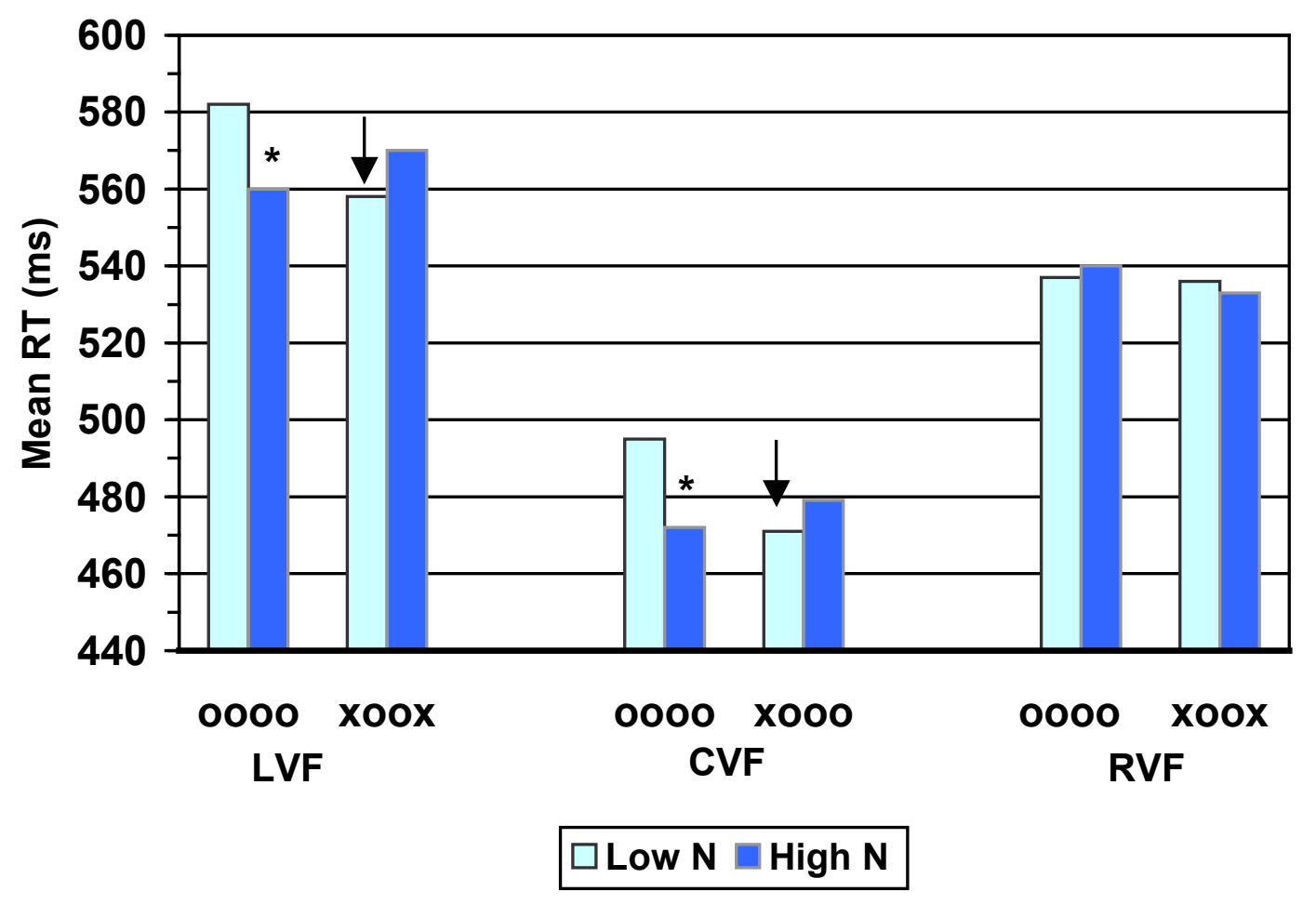

Figure 9: Experimental results for $\mathrm{CVF}$ and lateralized presentation of high-N versus low-N words. Presentation conditions and statistically significant differences are displayed in the same manner as Figure 8. 


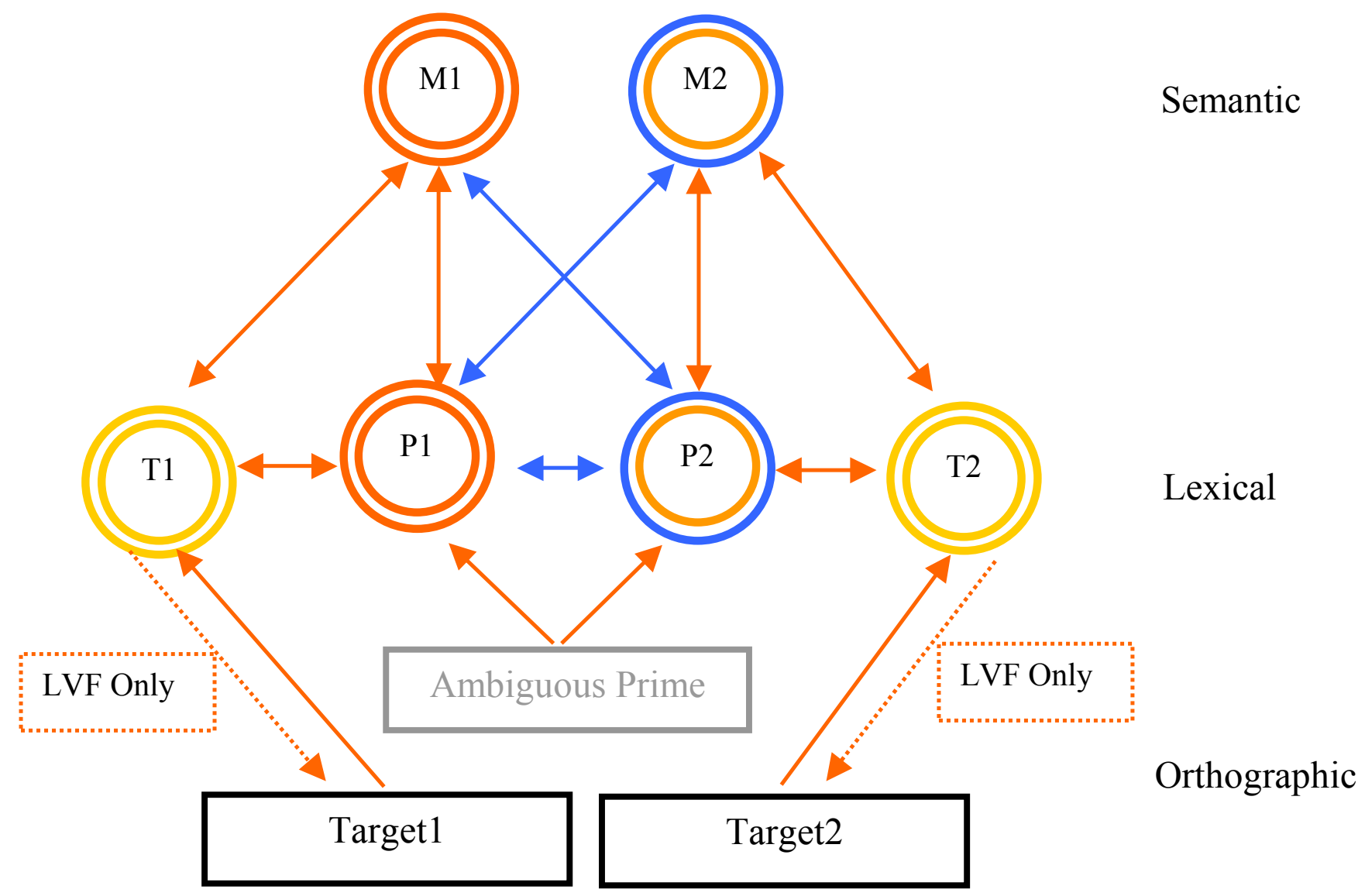

Figure 10: Illustration of proposed effect of $750 \mathrm{~ms}$ SOA in Burgess and Simpson (1988). Arrows represent net effect of interactions (orange = excitatory; blue $=$ inhibitory). Inner node colors reflect the state of activation approximately $350 \mathrm{~ms}$ after prime presentation (orange $=$ excited; blue $=$ inhibited). Outer node colors represent the state of activation just before presentation of the target. When the prime is presented, it initially excites both meanings $(\mathrm{P} 1, \mathrm{M} 1=$ dominant meaning; $\mathrm{P} 2, \mathrm{M} 2$ = subordinate meaning), and spreading activation results in partial activation of word nodes of the related targets (T1, T2). Over time, the dominant meaning inhibits that subordinate meaning, so that prior to target presentation, P2 and M2 are inhibited, but this inhibition does not directly affect T2. For a target related to the dominant meaning (Target1), the priming of $\mathrm{T} 1$ and $\mathrm{M} 1$ provide facilitation for presentation to either VF. For RVF presentation of a word related to the subordinate meaning (Target2) top-down facilitation of the orthographic encoding is not possible, and the lack of excitatory support from M2 slows down the rate of activation of T2, so the net effect is no facilitation. For LVF presentation of Target2, topdown input from $\mathrm{T} 2$ provides orthographic priming, so there is a net facilitatory effect. 


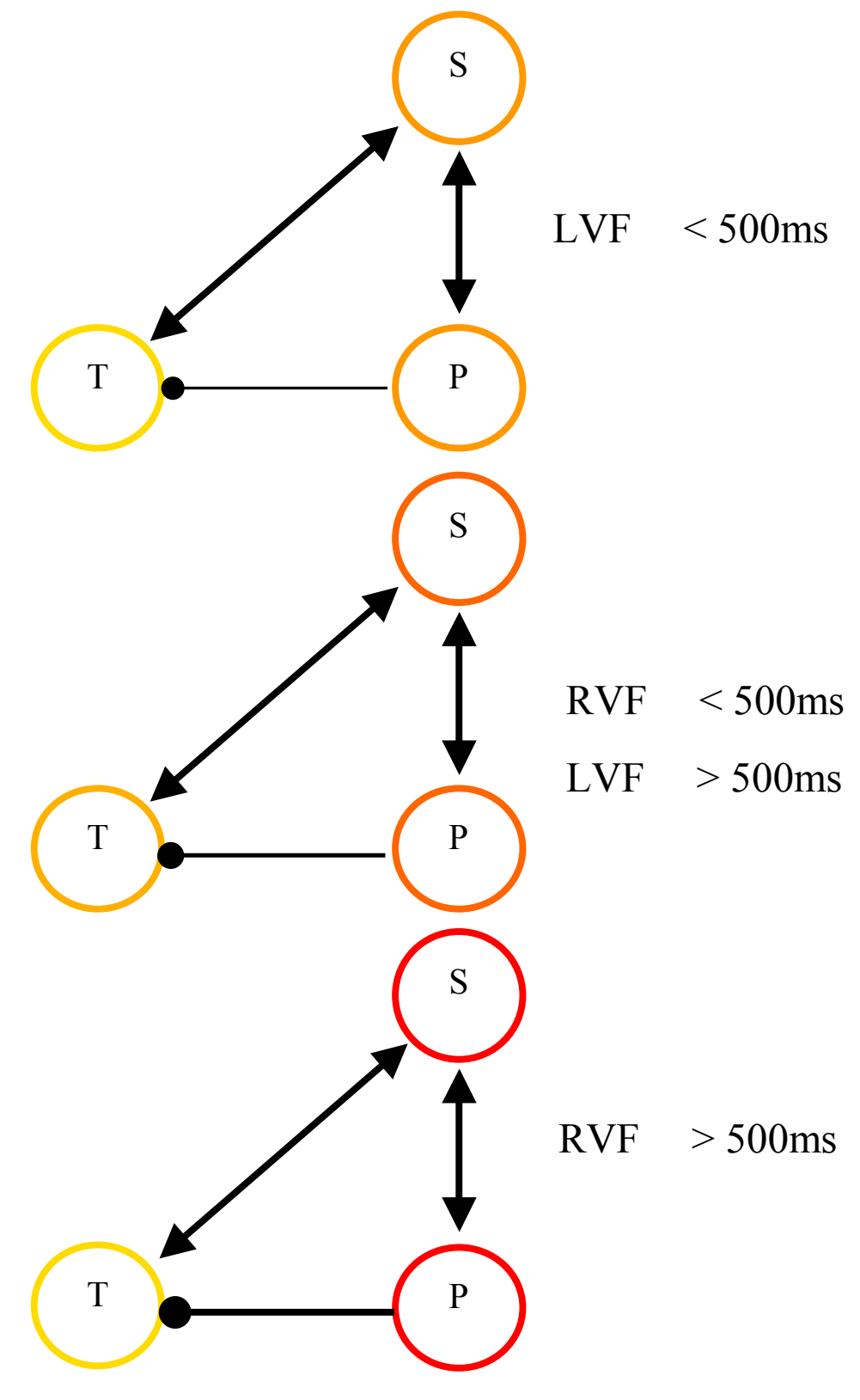

Figure 11: Proposed activation patterns for semantic-only primes by SOA and visual field. $\mathrm{P}$ and $\mathrm{T}$ denote lexical representations of the prime and target, respectively, while $\mathrm{S}$ represents semantic features common to both the prime and target. Activation level is depicted via the redness of the node (yellowish $=$ lowest activation; reddish $=$ highest activation). Excitatory connections are shown by arrows, while an inhibitory relationship is shown by a closed circle, where the width of the line indicates the net inhibitory effect. For SOAs of less than $500 \mathrm{~ms}$, the activation of the target increases with the activation of the prime. For SOAs of more than 500ms, RVF prime activation reaches a level in which increased inhibition outpaces increased excitation of the target word. 


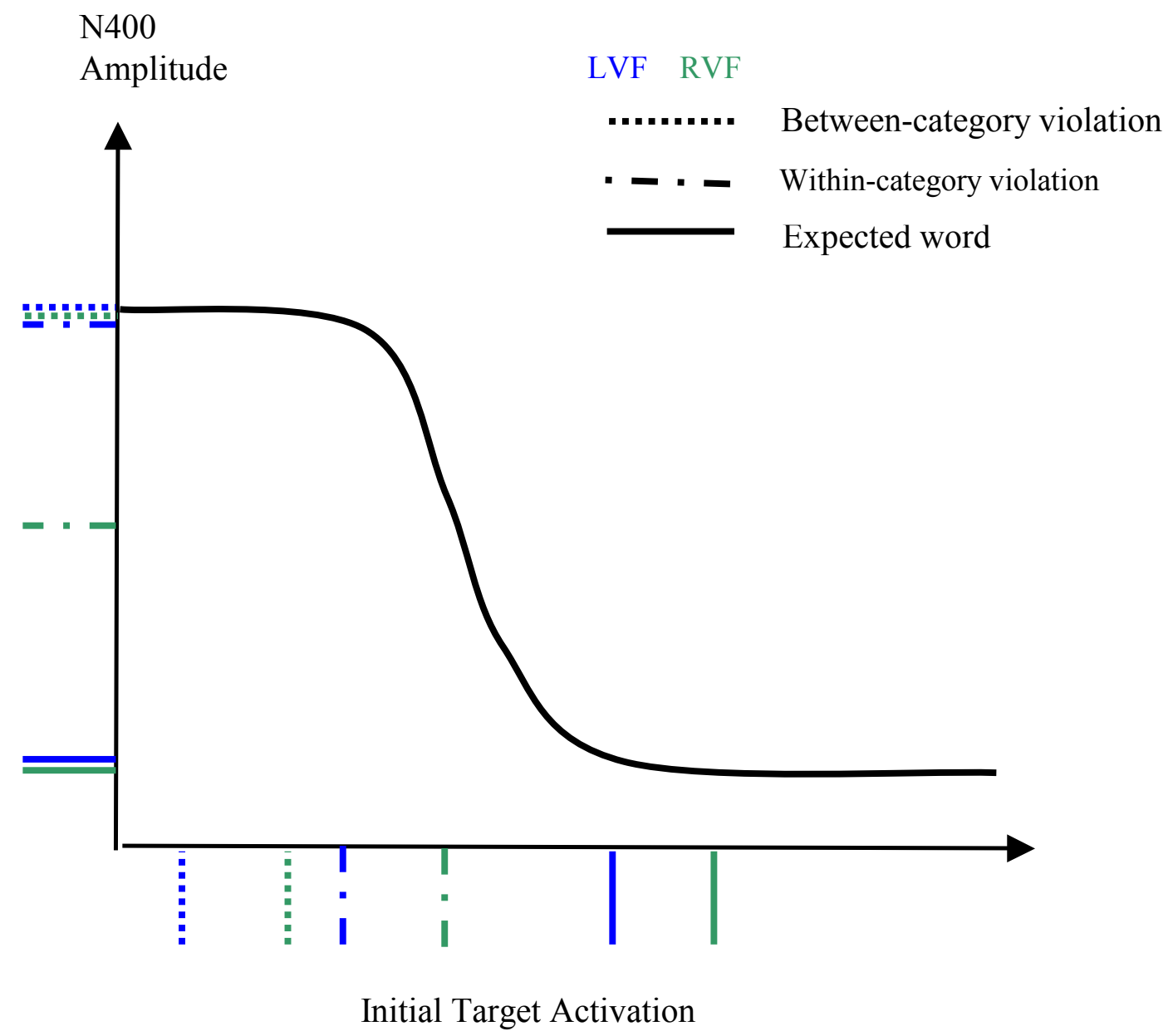

Figure 12: Illustration of proposed N400 patterns. The initial target activation for LVF presentation is reduced the same amount with respect to the corresponding RVF condition for all three types of target words. However, due to the shape of the N400 amplitude function, the resulting LVF N400 amplitude only differs from the corresponding RVF amplitude for the within-category violation. 
